\title{
Tailored Surface Engineering of Pigments by Layer-by-Layer Coating
}

\author{
Lars Dähne $^{a} \cdot$ Julia Schneider ${ }^{a} \cdot$ Dirk Lewe $^{b} \cdot$ Henrik Petersen ${ }^{b}$ \\ ${ }^{\text {a }}$ Surflay Nanotec GmbH, Berlin, and ${ }^{b}$ MT.DERM GmbH, Berlin, Germany
}

\begin{abstract}
We have evaluated the feasibility of layer-by-layer encapsulation technology for the improvement of dye pigments used for tattoos or permanent make-up. The formation of core-shell structures is possible by coating pigments with thin films of several different polyelectrolytes using this technology. The physicochemical surface properties, such as charge density and chemical functionality, can be reproducibly varied in a wide range. Tailoring the surface properties independently from the pigment core allows one to control the rheological behaviour of pigment suspensions, to prevent aggregation between different pigments, to reduce the cytotoxicity, and to influence the response of phagocytes in order to have similar or the same uptake and bioclearance for all pigments. These properties determine the durability and colour tone stability of tattoos and permanent make-up. @ 2015 S. Karger AG, Basel
\end{abstract}

\section{Introduction}

In recent times, the desire for tattoos and permanent make-up has increased tremendously [1]. Consequently, the variety and to some degree the safety of coloured dye pigments used in tattooing have broadened remarkably. Due to the increasing importance of pigments, scientific research on pigment safety, pigment improvement, and the mechanisms of tattoo durability as well as tattoo removal has started to evolve [2]. However, due to the wide range of different pigments available and the complicated interactions between these dyes and the dermis, based on the immune response of the skin, research on these problems is still at an early stage and requires further dedicated studies.

Several health issues related to embedding different pigments into the skin are already recognised, such as toxicity, especially for heavy-metal pigments; potential cancerous risk, e.g. for the polycyclic aromatic compounds in Carbon Black $[3,4]$ and in azo dyes; the slow dissolution of pigments; the photoreactivity of organic dyes; and the formation of dangerous radicals by titanium dioxide $\left(\mathrm{TiO}_{2}\right)$ pigments [5]. These problems arise mainly as a result of direct contact between the dangerous compound or its surfaces with the tissue of the dermis, leading to an immune response in the body. Besides the health risks, there are also several problems in the quality of tattooing, arising mainly from insufficient long-term stability. This leads to fading and shifts of colour tones over time, which often requires a refreshment of the pigment injection. For example, as our own observations have shown, brown colour tones based on 
Fig. 1. a Steps 1 to 4 show the process of the layer by layer coating for planar surfaces. In steps 1 and 3 either a positively or negatively charged polyelectrolyte is adsorbed to the surface. In steps 2 and 4 the surface is washed in order to remove excess polyion. Example polycation poly(allylamine hydrochloride) $(\mathrm{PAH}, \mathbf{b})$ and the polyanion poly(styrenesulfonate sodium salt) (PSS, c).

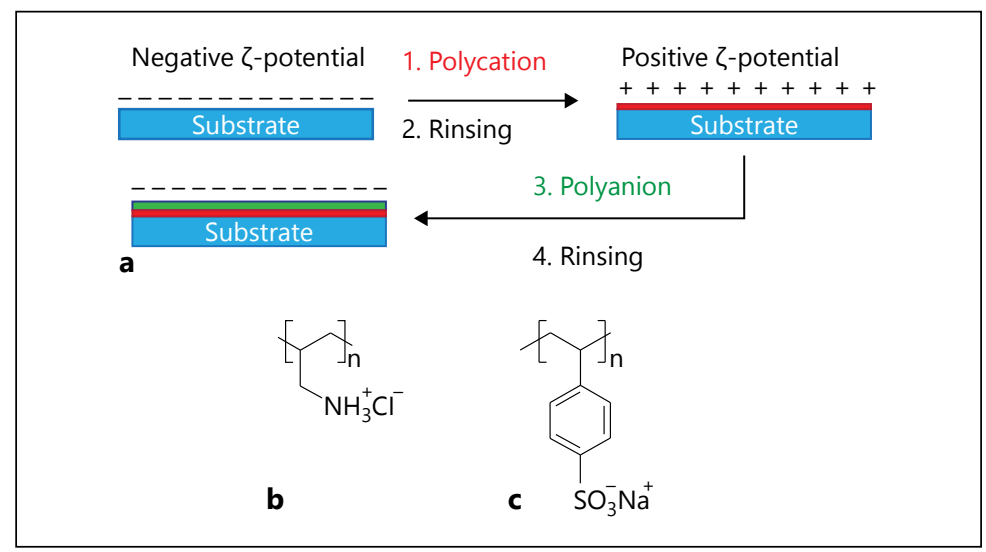

inorganic pigments containing different iron oxides for black, red, and yellow as well as $\mathrm{TiO}_{2}$ for white shift to a rose tone after several months due to a faster removal of yellow pigments compared with red and white ones. In contrast, for organic pigments, the colour shifts from warm brown to grey, caused by faster disappearance of red and yellow pigments. Several factors determine the instability of pigments in the skin, such as dissolution, chemical degradation, photobleaching, and enzymatic degradation or bioclearance by the phagocytes of the immune system. Phagocytes take up foreign particles according to recognition of the particles as being dangerous for the body. This recognition is based on the physicochemical surface properties and the size of the particles. After uptake of those particles, the phagocytes can leave the skin in order to remove the pigment from the organism, which leads to fading. Moreover, the cells could also encapsulate the pigments and remain in the skin, which would increase the long-term stability of the tattoos.

Some of the above-described problems can be reduced by encapsulating the pigments in polymer shells, thus preserving their size and colour but unifying and optimising their surface properties. This requires a thin, transparent, and homogeneous coating with a thickness in the nanometre range. A possible solution is encapsulation based on layer-by-layer (LbL) technology. This self-assembly process was developed on planar surfaces approximately 20 years ago [6]. Some years later it was extended to colloidal systems [7, 8]. Nowadays, LbL technology is the subject of intensive research on many different applications, such as corrosion control, biomedical applications, and material science applications, including drug delivery, catalysis, and medicine [9].

LbL technology allows for charged surfaces to be coated with polymer films and for the thickness of these films to be controlled on the nanometre scale. This is demonstrated by coating a pigment to generate a negatively charged surface, determined by electrophoresis as the $\zeta$-potential [10]. If an aqueous solution of a positively charged polyelectrolyte, such as poly(allylamine hydrochloride) (PAH) (fig. 1b), is applied to the pigment, the PAH will be adsorbed onto the surface due to electrostatic attraction and an increase in entropy. Not only are the negative charges on the pigment surface neutralised by the adsorption but also the $\zeta$-potential of the pigment surface is completely reversed to a positive value. When a defined positive potential is achieved, the adsorption stops due to repulsion of further $\mathrm{PAH}$ molecules in the solution. After rinsing, excess $\mathrm{PAH}$ is removed, and the process can be repeated by assembling a polyanion, such as 


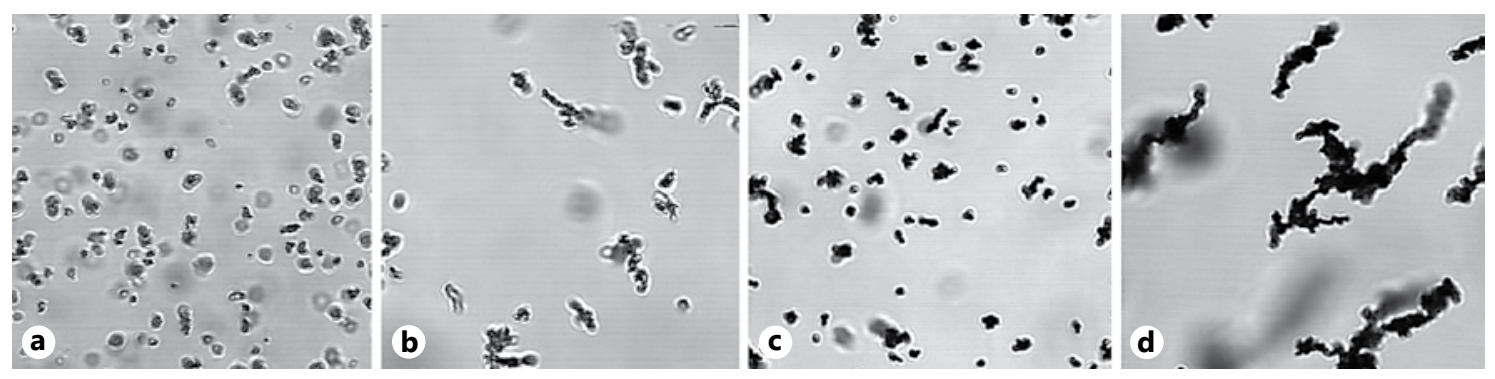

Fig. 2. Transmission microscope images of inorganic tattoo pigments (image size $40 \mu \mathrm{m} \times 40 \mu \mathrm{m}$ ). a Titanium dioxide; b iron oxide yellow; c iron oxide red; $\mathbf{d}$ aggregated iron oxide black.

poly(styrenesulfonate sodium salt) (PSS; fig. 1c), in the same manner, which changes the surface potential back to a negative value (fig. 1a). This can be repeated many times, yielding a constant increase in thickness of approximately $4 \mathrm{~nm}$ for each double layer of PAH/PSS. A variety of more than 50 polyanions and also polycations with quite different properties and functionalities are commercially available, giving this technology enormous potential to easily modify the surface properties of particles.

We will report pigment encapsulation in $\mathrm{LbL}$ films and present initial results regarding the properties of such core-shell structures and their potential for future applications in the tattoo and permanent make-up industry. Although LbL coating can be applied to all pigments, we will focus on the encapsulation of inorganic tattoo pigments, including $\mathrm{TiO}_{2}$, iron oxide black, iron oxide yellow, and iron oxide red.

\section{Experimental Section}

\section{Physicochemical Properties of Selected Pigments}

Pigments are characterised by properties such as chemical composition, colour, size distribution, shape, porosity, surface roughness, and surface charge. The last is the determining property for pigment encapsulation. Thus, the surface charges of some tattoo pigments were determined; these
Table 1. ל-Potentials of dye pigments used in tattoo and permanent make-up applications determined in $10 \mathrm{~mm}$ Tris buffer solution at $\mathrm{pH} 7$ by electrophoresis using a Zetasizer from Malvern Instruments

\begin{tabular}{ll}
\hline Tattoo pigment & 乙-Potential in $\mathrm{mV}$ \\
\hline Titanium dioxide & -45.0 \\
Iron oxide yellow & -29.9 \\
FD\&C Yellow 6 Lake & -18.2 \\
DSC Red 30 Lake & -30.1 \\
Pigment Red 5 & -25.1 \\
Iron oxide red & -32.0 \\
FD\&C Red 40 Lake & +21.7 \\
Pigment Blue 15 & -30.3 \\
Iron oxide black & -29.3 \\
Carbon black & -24.9
\end{tabular}

are listed in table 1. Apart from the positively charged FD\&C Red 40 Lake, all of them exhibit a negative surface charge. High-resolution confocal laser scanning microscopy (CLSM) has been used for analysing size distribution, porosity, and polyelectrolyte coating on the surface by means of fluorescently labelled polyelectrolytes. Transmission images of tattoo pigments (fig. 2) show that the size varies between 1 and $10 \mu \mathrm{m}$. There are multiple shapes, from almost spherical to needlelike. In the case of iron oxide black, the ferromagnetic properties can be detected as causing the formation of temporary aggregates between the primary pigment particles. 
Fig. 3. Progress of surface charge of tattoo pigments with increasing number of surface layers. The $\zeta$-potential becomes positive after adsorption of the first polycationic PAH layer. The adsorption of a second polyanionic PSS layer turns the potential back to negative, and so forth.
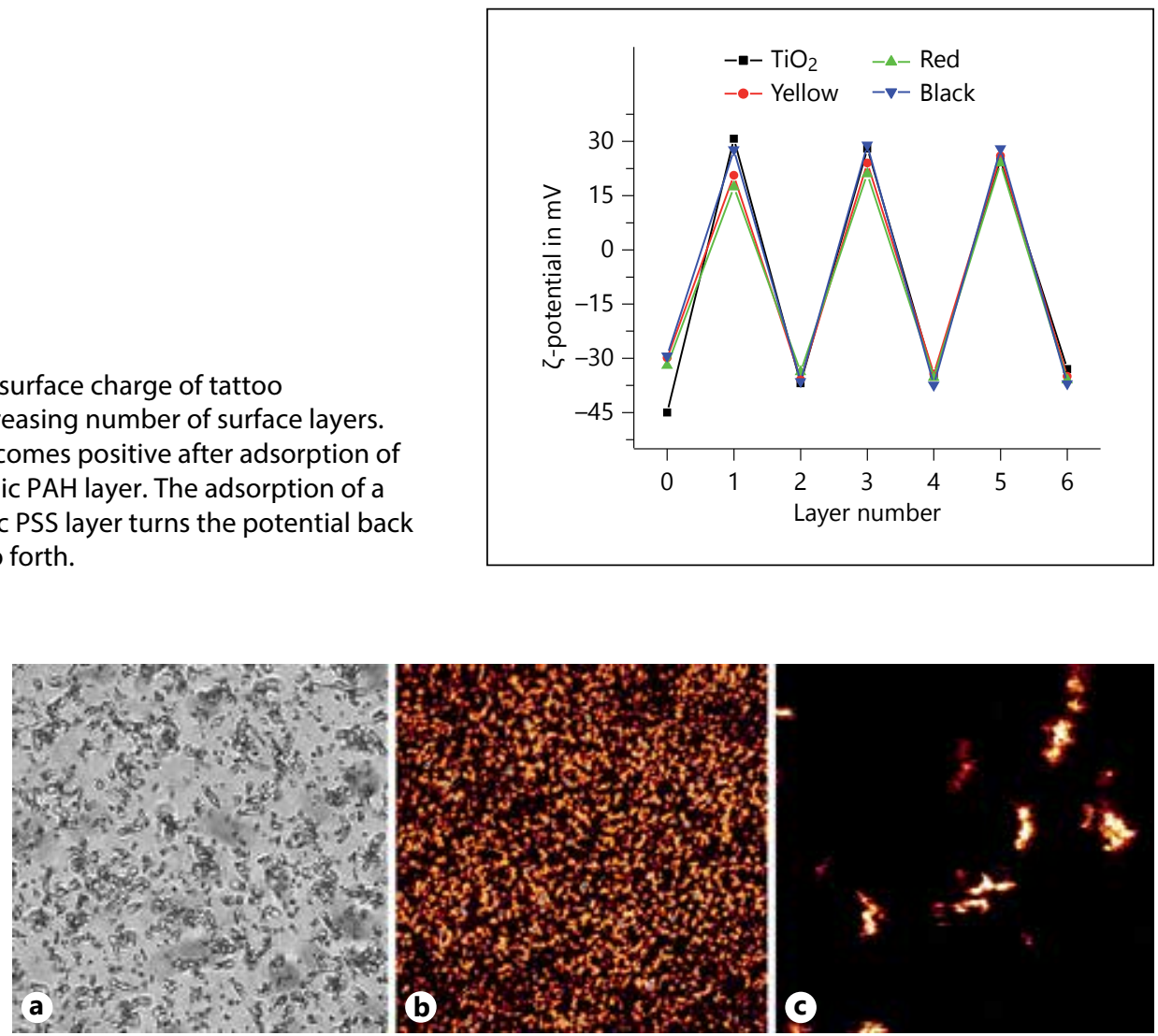

Fig. 4. Transmission and confocal laser scanning microscopy (CLSM) fluorescence images of (PAH-Rho/PSS) ${ }_{2}$-coated iron oxide yellow: a transmission image; b CLSM fluorescence image $(80 \times 80 \mu \mathrm{m})$; c high-resolution CLSM fluorescence image $(20 \times 20 \mu \mathrm{m})$.

Physicochemical Properties of Layer-by-Layer Coatings

The four different pigments were LbL coated with 6 layers, starting with the polycation $\mathrm{PAH}$ and then alternating PSS and PAH. One way to control coating success is to measure the change in the $\zeta$-potential (fig. 3). After the first layer, the charge changes to a positive value for all pigments. Following deposition of the next layer (PSS), the charge changes back to a negative value, and so on. While the surface charges of different pigments are different at the beginning, they become more equal as the layer number and film thickness increase.
The coated pigments exhibit almost no change in size compared with the uncoated ones because the layer thickness after 6 layers is around $12 \mathrm{~nm}$, which is negligible in comparison to the particle diameter.

The polymer film is not visible by transmission microscopy due to the limited spatial resolution of optical microscopes. However, fluorescently labelled polyelectrolytes help to visualise the deposited polyelectrolytes in the fluorescence images of a CLSM (fig. 4). The fluorescence intensity is correlated with the adsorbed amount of polyelectrolytes. The pigments were coated with two double layers of PSS and PAH, 


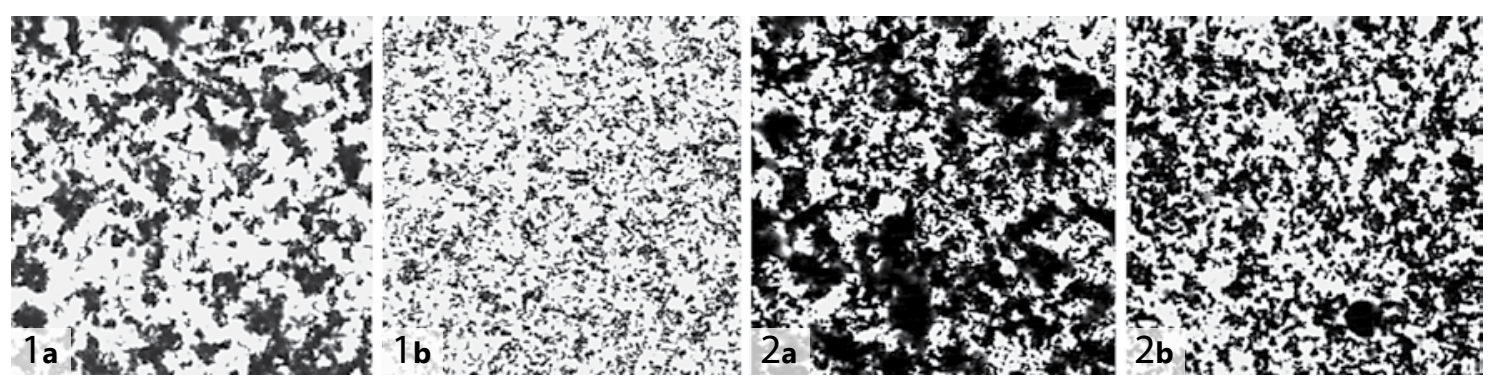

Fig. 5. Microscopy images of uncoated and coated pigments: (1) iron oxide yellow, (2) iron oxide red; a uncoated and b LbL-coated particles (PAH/PSS) ${ }_{2}$; image size $80 \times 80 \mu \mathrm{m}$.

Fig. 6. Percentage of surviving RKO cancer cells after $24 \mathrm{~h}$ incubation with uncoated or coated (PAH/PSS/ $\mathrm{PAH} /$ Polymer $\mathrm{X}$ ) titanium dioxide and silicon dioxide particles whereupon the external coating was varied $(X=P S S$, poly(methacrylic acid sodium salt PMAA), Nafion, or poly(vinyl phosphate sodium salt)PVPho). The tests were performed at two different particle concentrations.

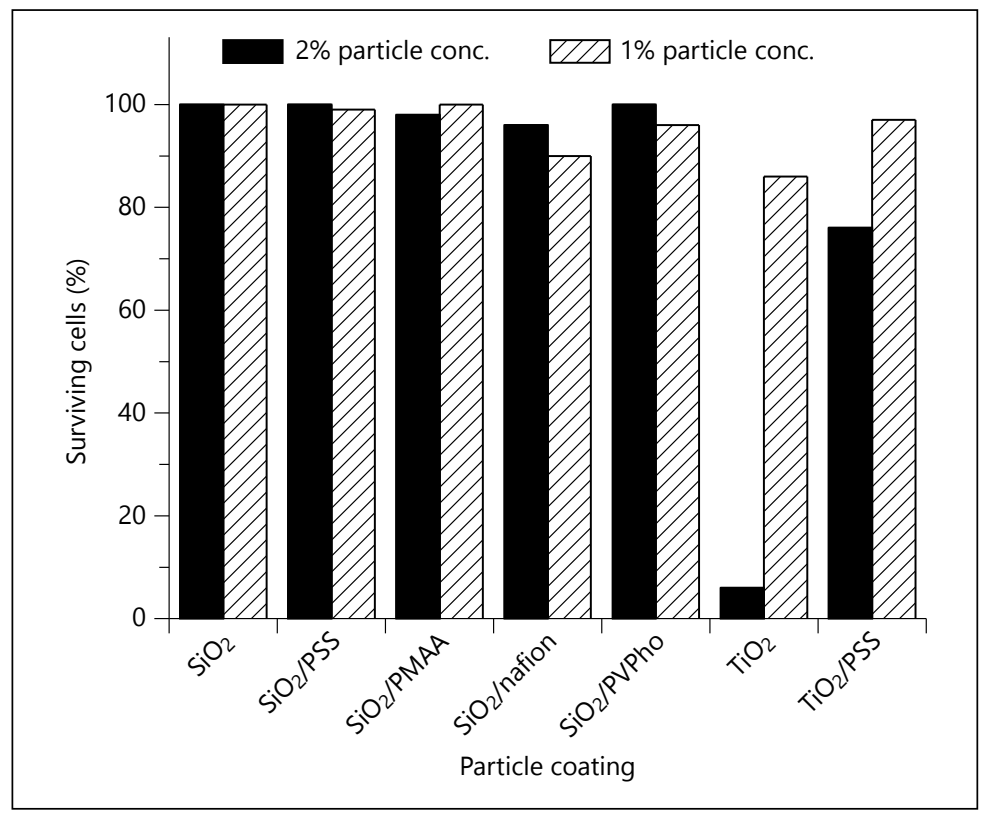

with PAH covalently linked to the fluorescent dye rhodamine B (PAH-Rho) at every 160th monomer position. Figure $4 \mathrm{c}$ shows some fluorescence in the interior of the particles, indicating the deposition of PAH-Rho inside the pores or fissures of the particles.

The deposition of LbL films on different pigment particles leads to homogeneous, chemically identical surfaces with a uniform $\zeta$-potential, independent of the core material. Furthermore, the external surface potential can be tuned to be either negative or positive, depending on the polyelectrolyte species of the last layer.

The unification of and partial increase in the surface charge of pigments increase their repulsion and diminishes the tendency to aggregate during storage. Aqueous suspensions of uncoated and coated iron oxide yellow and red were sonicated and stored for 3 days. After shaking, CLSM images of the samples showed different size distributions for the particles (fig. 5). The uncoated particles showed a much higher degree of aggre- 
Fig. 7. Phagocytosis of LbL-coated silica particles with a $4.3 \mu \mathrm{m}$ diameter. In addition to the percentage of ingested particles (black), the percentage of sacrificed phagocytes (patterned) forming DNA nets is shown. The $x$-axis indicates the external polyelectrolyte layer. Phosphate buffered saline (PBS) and Escherichia coli are the negative and positive control samples.

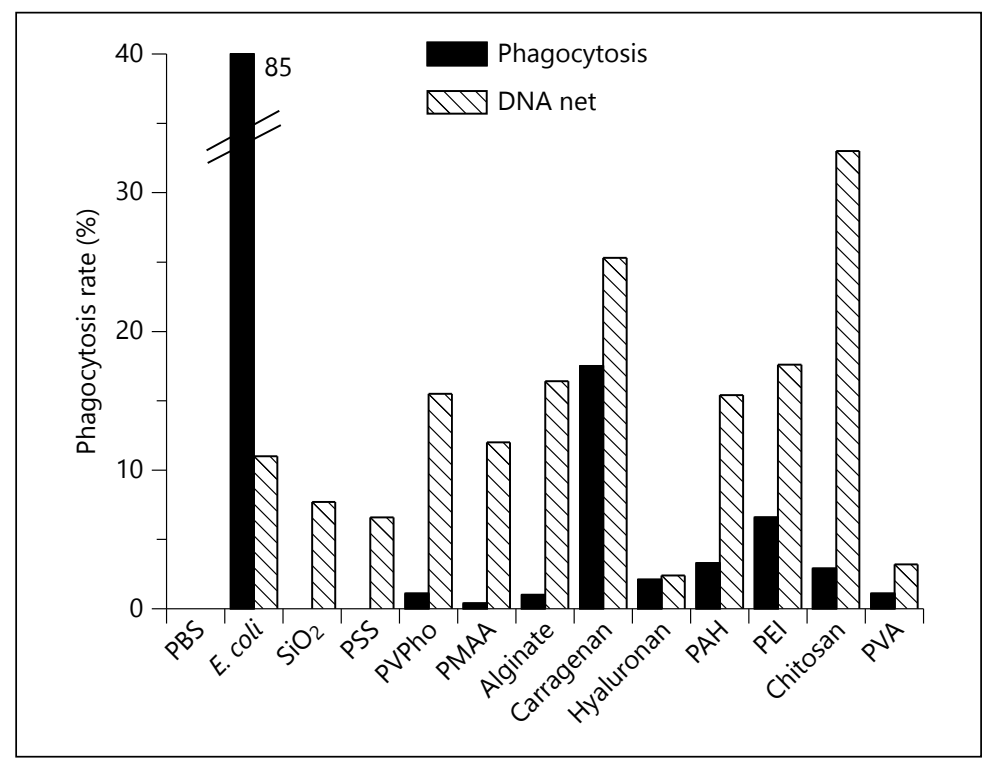

gation when compared with the coated ones. This phenomenon is even more noticeable for mixtures of different uncoated pigments, sometimes yielding strong aggregation due to large differences in their $\zeta$-potentials. However, mixtures of coated pigments with different gravimetric densities show the undesired effect of faster phase separation. As a second consequence suspensions of coated particles can have a concentration 1.5 times higher than uncoated ones and still have the same liquid properties.

\section{Cell Response to Layer-by-Layer Coated Particles} The equalisation of the surface properties is expected to have remarkable effects on the behaviour in the skin. Thus, initial tests have been performed in order to investigate the cytotoxicity of different LbL coatings, as well as their recognition by human phagocytes. For this purpose, different polyelectrolyte coatings have been chosen and assembled on monodisperse model particles, including silicon dioxide $\left(\mathrm{SiO}_{2}\right)$ and titanium dioxide $\left(\mathrm{TiO}_{2}\right)$. Particles of $1 \mu \mathrm{m}$ in diameter were used for cytotoxicity tests and $\mathrm{SiO}_{2}$ particles with $4.3 \mu \mathrm{m}$ for phagocytosis tests. The particles were coated with a basic layer of PAH/PSS/PAH, while the last layer was varied using different polyanions, such as poly(methacrylic acid sodium salt) (PMAA); Nafion, which is sulphonated Teflon; or poly(vinyl phosphate sodium salt) (PVPho). The cytotoxicity test was performed with RKO cancer cells that were incubated for $24 \mathrm{~h}$ in 1- or 2-percent-by-weight particle suspensions. The incubation was performed under very slow agitation in order to avoid particle sedimentation on the cells as well as abrasion of the cells by the moving particles. The results are shown in figures 6 and 7.

The investigations with $1 \mu \mathrm{m} \mathrm{SiO}_{2}$ particles showed that despite the high particle concentration, the PSS- and PMAA-coated particles are well tolerated by the cells. The hydrophobic $\mathrm{Na}$ fion and the PVPho coatings revealed a slightly decrease of the cell tolerance (fig. 6). In contrast, uncoated $\mathrm{TiO}_{2}$ showed a clear toxic effect that could be dramatically reduced by the encapsulation with PAH/PSS. The surprisingly high cytotoxicity of $\mathrm{TiO}_{2}$ could have been caused by radical formation on its surface by light irradiation, which could not be fully avoided during the ex- 
periment. In addition, the high density of $\mathrm{TiO}_{2}$, $4.3 \mathrm{~g} / \mathrm{cm}^{3}$, compared to $1.8 \mathrm{~g} / \mathrm{cm}^{3}$ for $\mathrm{SiO}_{2}$, could have led to more sedimentation and additional mechanical stress on the cells.

The experiments with human phagocytes were performed using $4.3 \mu \mathrm{m} \mathrm{SiO} 2$ particles with different LbL coatings. In the standard phagocytosis tests, the number of particles ingested by the phagocytes is correlated with their danger potential [11]. In addition to phagocytosis for some particles, we observed sacrificed phagocytes that released their DNA in order to immobilise the particles with a DNA net. The results summarised in figure 7 show both the percentage of ingested particles and the number of sacrified phagocytes.

The results reveal that by LbL coating, the response of the immune system to particles can be strongly influenced. For instance, positively charged surfaces (PAH, polyethylenimine (PEI), and chitosan in fig. 7) showed greater phagocytosis than the negative ones (PVPho, PSS, and hyaluronan), which is in good agreement with the literature [9]. Surprisingly, the biopolymers alginate and carrageenan resulted in greater phagocytosis compared with the synthetic polymer PSS and polyvinyl alcohol (PVA).

Since the unusual DNA-net formation was assumed to be eventually caused by the large size of the particles, further investigations on smaller LbL coated particles as well as on real tattoo pigments are in progress.

\section{Summary and Outlook}

We have evaluated the feasibility of LbL encapsulation technology for the improvement of tattoo and permanent make-up pigments. It was shown that formation of core-shell structures is possible by coating pigments with thin films composed of several different polyelectrolytes. An overview of possible problems in tattooing and the possibility of addressing these problems with LbL technology is given in figure 8 .

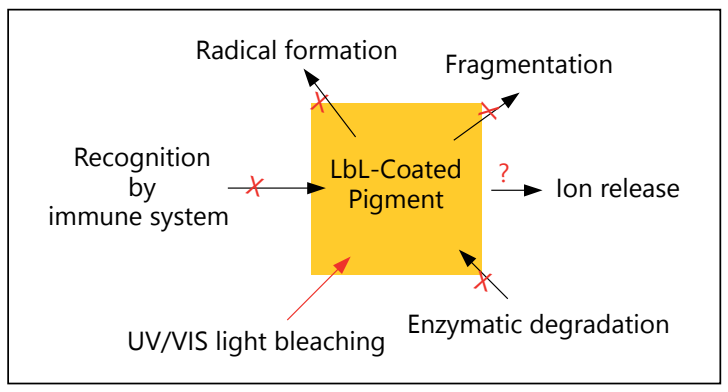

Fig. 8. Processes that are considered of importance for tattoos. The red crosses show processes that can be potentially prevented by LbL encapsulation, while photobleaching and slow dissolution probably cannot be inhibited by this method.

Physicochemical surface properties such as charge density and chemical functionality could be reproducibly varied in a wide range. The new surface properties changed the rheological behaviour of pigment suspensions remarkably, preventing aggregation between different pigments but also resulting in faster phase separation of pigment mixtures. Investigation of the response of cells and phagocytes to coated model particles showed that LbL films have the potential to reduce or even prevent the fading and shifts of colour tones of tattoos caused by phagocytosis.

Additionally, the polymer network around the pigments can prevent their fragmentation into smaller pieces. Beneficial is also the very thin and transparent polyelectrolyte shell that does not change the colour of the pigments by absorption of visible light. However, it does not efficiently absorb UV light to protect them against photobleaching. From numerous studies of the permeability behaviour of polyelectrolyte films, the following possible advantages can be derived. First, LbL polyelectrolyte films are impermeable to macromolecules such as enzymes [8], which should prevent enzymatic degradation of coated pigments. Second, the release of dissolved molecules from the pigment surface can be reduced or completely stopped if the molecules are highly charged or have a high 
molecular weight. Third, recent investigations on LbL membranes showed that oxygen transport is strongly hindered [12], which could also prevent chemical oxidation of pigments. Fourth, radical formation on encapsulated pigment surfaces by photochemical processes [5] will not affect human tissue cells but will instead be quenched by the polyelectrolyte shell.

\section{Acknowledgements}

We are grateful to Dr. Radostina Georgieva and Prof. Hans Bäumler (Institute of Transfusion Medicine, Charité Berlin) for the phagocytosis tests. For the cytotoxicity tests, we thank Dr. Manfred Jugold (Deutsches Krebsforschungszentrum Heidelberg). Many thanks also to Barbara Baude for the experimental work.

\section{References}

1 Bäumler W: The fate of tattoo pigments in the skin (presentation). First International Conference on Tattoo Safety, Berlin, 2013.

2 Luch A, Laux P, et al: Tattoos: is there more than 'meets-the-eye'? Tattooing from a toxicological perspective. The Lancet, submitted.

-3 Sahu D, Kannan GM, Vijayaraghavan R: Carbon black particle exhibits size dependent toxicity in human monocytes. Int J Inflam 2014;2014:827019.

4 Lehner K, Santarelli F, Vasold R, König B, Landthaler M, Bäumler W: Black tattoo inks are a source of problematic substances such as dibutyl phthalate. Contact Dermatitis 2011;65:231-238.
>5 Rancan F, Nazemi B, Rautenberg S, Ryll M, Hadam S, Gao Q, Hackbarth S, Haag SF, Graf C, Rühl E, Blume-Peytavi U, Lademann J, Vogt A, Meinke MC: Ultraviolet radiation and nanoparticle induced intracellular free radicals generation measured in human keratinocytes by electron paramagnetic resonance spectroscopy. Skin Res Technol 2014;20: 182-193.

6 Decher G, Hong JD, Schmitt J: Buildup of ultrathin multilayer films. Thin Solid Films 1992;210:831-835.

7 Donath E, Sukhorukov GB, Caruso F, Davis SA, Möhwald H: Novel hollow polymer shells by colloid-templated assembly of polyelectrolytes. Angew Chem Int Ed 1998;41:4019-4022.

$>8$ Peyratout C, Dähne L: Tailor-made polyelectrolyte microcapsules: from multilayers to smart containers. Angew Chem Int Ed 2004;43:3762-3783.
-9 Mercato LL, Rivera-Gil P, Abbasi AZ, Ochs M, Ganas C, Zins I, Sönnichsen C, Parak WJ: LbL multilayer capsules: recent progress and future outlook for their use in life sciences. Nanoscale 2010;2:458-467.

$10 \mathrm{http} / /$ www.silver-colloids.com/ Tutorials/Intro/pcs1.html (accessed 2001-2012).

11 Prietl B, Meindl C, Roblegg E, Pieber TR, Lanzer G, Fröhlich E: Nano-sized and micro-sized polystyrene particles affect phagocyte function. Cell Biol Toxicol 2014;30:1-16.

12 Jang WS, Rawson I, Grunlan JC: Layerby-layer assembly of thin film oxygen barrier. Thin Solid Films 2008;516: 4819-4825. 